\title{
IoT Mining Tracking \&Worker Safety Emergency Alert
}

\section{S Aruna Kumari ${ }^{1} \mid$ K Hamsika ${ }^{2}$ | G Sandya ${ }^{2}$ | V Purushottam Rao ${ }^{2}$ | P Sai Kumar ${ }^{2}$}

${ }^{1}$ Assistant Professor, Department of Electronics and Communication Engineering, Raghu Institute of Technology, Visakhapatnam, India.

2UG Student, Department of Electronics and Communication Engineering, Raghu Institute of Technology, Visakhapatnam,

\section{To Cite this Article}

S Aruna Kumari, K Hamsika, G Sandya, V Purushottam Rao and P Sai Kumar, "IoT Mining Tracking \&Worker Safety Emergency Alert", International Journal for Modern Trends in Science and Technology, Vol. 06, Issue 06, June 2020, pp.:138-141; https://doi.org/10.46501/IJMTST060628

\section{Article Info}

Received on 15-May-2020, Revised on 01-June-2020, Accepted on 10-June-2020, Published on 19-June-2020.

\section{ABSTRACT}

Mining is indispensable to the creation of goods, infrastructure and services which enhance the quality of their lives. Demand of coal as energy resources is always important and significant. But thousands of people have lost their lives in mining accidents, all over the world working in the earth presents many different security and health dangers. So here we propose a mining tracking as well as safety system for the mining industry using microcontroller based circuit on the worker safety. We use RF based circuitry to detect workers moving through the entire mining site. We used ARDUINO UNO as main micro controller. The system makes use of ATMEGA Microcontroller based RF tracker circuitry used to transmit and receive data by using EM-18 RFID reader module with RFID cards. This helps to track the current location of workers through the entire mining site. Moreover each RFID card circuit is integrated with a panic/emergency button. When this button is pressed it shows an emergency alert over the IOT webpage. This can be used for any emergencies like - toxic gas inhalation, physical injury etc. Thus the system ensures mining worker Safety using IOT.

KEYWORDS: ARDUINO UNO, ATMEGA 328P Micro controller, RF modules

Copyright (C) 2014-2020 International Journal for Modern Trends in Science and Technology

DOI: https://doi.org/10.46501/IJMTST060628

\section{INTRODUCTION}

The raising global population is putting together unavoidable issues. Mining are among the most important of those issues, within the area mining industries has a lot work to do. Some of them are fixed through GSM, GPS with different features, by using various kinds of sensors that can detect whether the worker is in danger or not. With ever growing technology, the advances are rising in the face of human- life causing situations. The mining industry merges everyday as a concept which is created a new branch as an alternative of human factor with various kinds of resuce systems. The components and working of this prototype are discussed in Sec.2.

\section{LITERATURE SURVEY}

A prototype consists of EM-18 RFID Reader Module and RFID card which work as a reader to read $125 \mathrm{KHz}$ tags is an inexpensive solution for an RFID based application. The reader comes with on- chip antenna and be powered up with a $5 \mathrm{v}$ supply power up module connect the transmit pin of the module to receive pin of your microcontroller. Radio-frequency identification uses electromagnetic fields to automatically identify and track tags attached to objects the tag 
transmits digital data, usually an inventory number back to the reader incase of sensors we are using Em-18 module and RFID card rather than using the GPS and GSM further this prototype can be advanced using different sensors which can be replaced by RFID Module. We can also use the GPS module to track the position of the miner it provides user with location and it gets calibrated by its own and it can be used by anyone but GPS accuracy depends on sufficient received signal quality. Zigbee technology is a wireless protocol used for wireless networks and connectivity it supports transfers of simple data like from sensors it supports low data rate it used for two-way communication between sensors and control system but it is a short distance communication like Bluetooth. In this project we avoided using zigbee due its cost effective and low data speed and it is not secured so we are replacing zigbee with ESP 8266 WIFI Module it has high power supply which makes it very easy to flash the board. The easiest development is the Arduino IDE it has ability to use the power jack as a power supply but there is no build wifi because most of projects related to IOT. We use IOT in almost all projects it is good board for starters in future that you buy ARDUINO version is WIFI included.

\section{PROPOSED WORK}

This project gives information about the mishap in the underground mines for worker safety. It consists of technical process of microcontroller unit we need establish hardware and need to install Arduino uno software to complete the project. When the system is turned ON, the microprocessor is triggered and the quest begins. DC power supply of $12 \mathrm{~V}$ is supplied to the regulator with the help of battery. Regulator acts as a medium between battery and microcontroller unit.

To operate this prototype model there should be a communication in between the system and the operator. So ATMEGA microcontroller is interfaced. Pair of two-channel are connected to the microcontroller when a worker press the push button is detected on either side, a command is sent to the end-users IOT webpage via the HT12E, HT12D. When the user receives the alert, the micro-controller is sent an ASCII action order. The message alert will be displayed on IOT webpage.

\section{BLOCK DIAGRAM}

Each block of system architecture is discussed in detail below

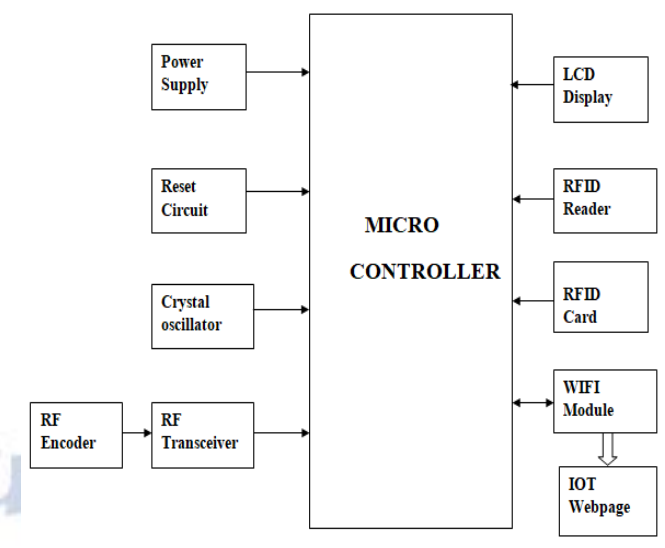

Fig-1: Architecture of Microcontroller

Any invention of latest technology cannot be activated without the source of power. So in this fast moving world we deliberately need a proper power source which will be apt for a particular requirement. All the electronic components starting from diode to IC's only work with a DC supply ranging from $5 \mathrm{~V}$ to $12 \mathrm{~V}$. Battery is used as a power supply medium.

The Arduino UNO is a microcontroller board based on the ATmega16U2. It has 14 digital input/output pins of which 6 can be used as PWM outputs, 6 analog inputs, a $16 \mathrm{MHz}$ crystal oscillator, a USB connection, a power jack and a reset button. It contains everything needed to support the microcontroller simply connect it to a computer with a USB cable or power it with a AC-to-DC adapter or battery to get started.

The board can operate on an external supply of 6 to $20 \mathrm{~V}$. It supplied with less than $7 \mathrm{~V}$, however the $5 \mathrm{~V}$ pin may supply less than five volts and the board may be unstable. The ATmega 328 has $32 \mathrm{~KB}$ of flash memory for storing code. It also has $2 \mathrm{~KB}$ of SRAM and $1 \mathrm{~KB}$ of EEPROM.

Radio frequency Identification (RFID) is a wireless identification technology that uses radio waves to identify the presence of RFID tags. Just like Bar code reader, RFID technology is used for identification of people, object. In barcode technology, we need to optically scan the barcode by keeping it in front of reader, whereas in RFID technology we just need to bring RFID tags in range of readers. Also, barcodes can get damaged or unreadable, which is not in the case for most of the RFID. It is used in many companies to provide access to their authorized employees.ESP8266 WIFI Module is a low-cost microchip with WI-FI enabled system on chip (SOC) module. It is mostly used for development of IOT embedded applications. It employs a 32-bit RISC CPU based system. It is a wireless transceiver that can be used 
end- point for IOT developments. ESP8266 is a 3V WIFI module very popular for its IOT applications. Maximum working voltage for $3.6 \mathrm{~V}$. It is very user friendly to provide internet connectivity to your projects.

Radio -Frequency identification (RFID) uses electromagnetic fields to automatically identify and track tags attached to objects. An RFID tag consists of tiny radio transponder, a radio receiver and transmitter. When triggered by electromagnetic interrogation pulse from a nearby RFID reader device, the tag transmits digital data usually an identifying inventory number, back to the reader. Active tags are powered by battery and thus can be read at a greater range from RFID reader unlike barcode, the tag doesn't need to be within line of sight of the reader . RFID is one method of automatic identification and data capture (ADIC). RFID tags are used in many industries.

A push-button is a simple switch mechanism to control some aspect of a machine or a process. Buttons are typically made out of hard material, usually plastic or metal. The surface is usually flat or shaped to accommodate the human finger or hand, so as to be easily depressed or pushed.

The HT12E Encoder ICs are series of CMOS LSIs for Remote Control system applications. They are capable of Encoding 12 bit of information which consists of address bits and 4 data bits. Each address and data input is externally programmable or fed in using switches. The HT12D Decoder ICs are series of CMOS LSIs for Remote Control system applications. These ICs are paired with each other. For proper operation a pair of encoder/decoder with the same number of address and data format should be selected (HT12E is paired with HT12D). The Decoder receive the serial address and data, transmitted by a carrier using an RF transmission medium and gives output to the output pins after processing the data.

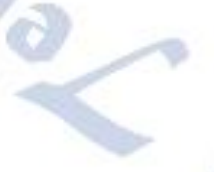

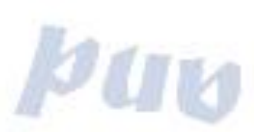

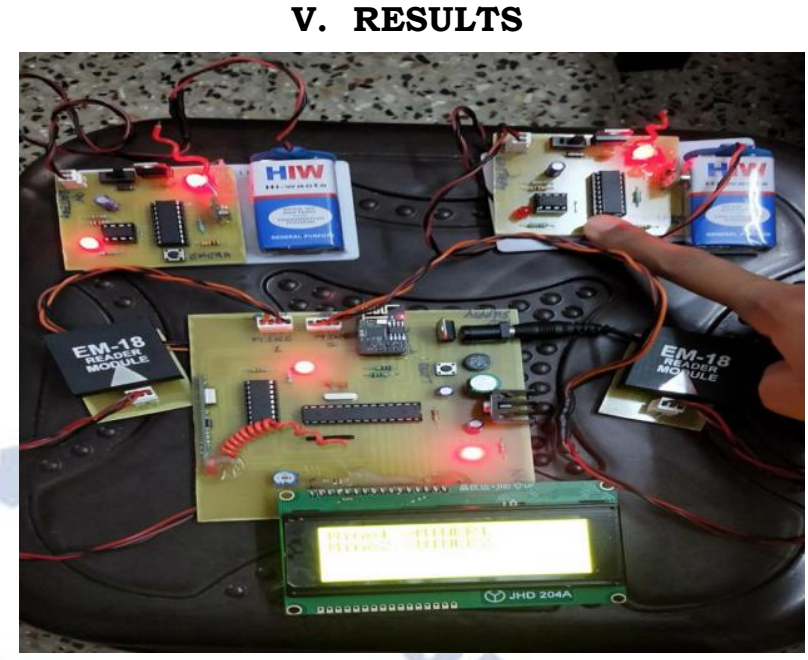

Fig 5.1(a) Miner-1 at the right side

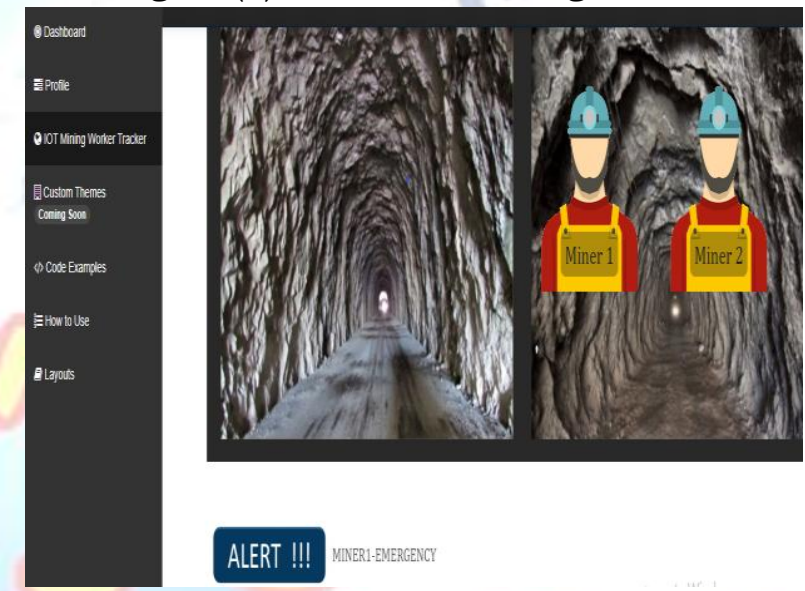

Fig 5.1(b) Emergency alert at miner-1

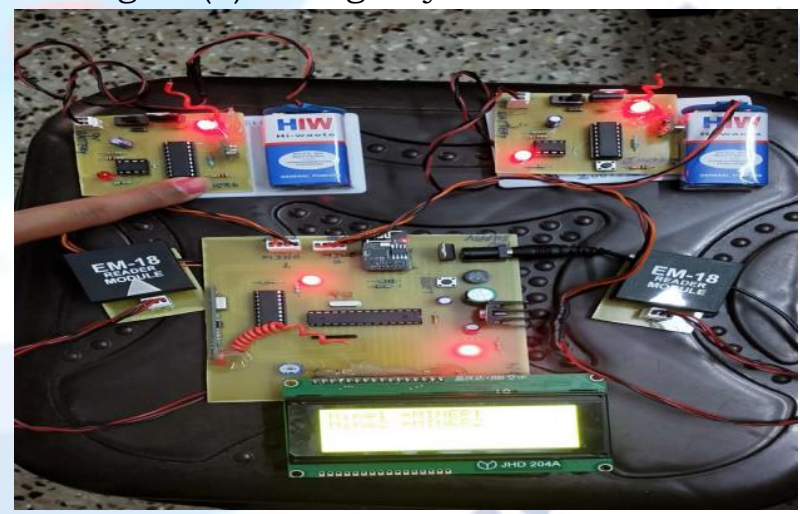

Fig 5.2(a) Miner-2 at the Left side

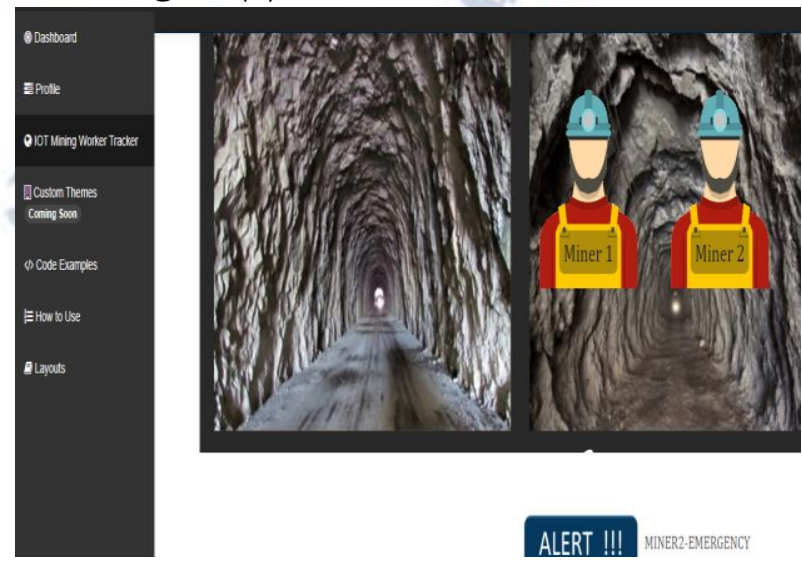

Fig 5.2(b) Emergency alert at miner-2 


\section{CONCLUSION}

The design and implementation of a system which can provide security for miner workers and take the appropriate action are required. The proposed method is used for beneficial of security purpose and industrial purpose. The work has been motivated by the desire to design a system that can detect danger and perform the required actions. This provides us the opportunity to pass on to alert to do the tasks that traditionally humans had to do but was life threatening. It can be enhanced by interfacing it with ATMEGA 328P Microcontroller and RFID reader module so that the person at the webpage can monitor the worker at anytime, anywhere.

\section{FUTURE SCOPE}

The project is expected to deployed on a global scale. It is motivated by desire to detect the dangers caused in underground mining for mining workers. In future prototype this further implemented to recognize various kinds of unsafe situation in mining industry for workers like carbon monoxide gas accumulation, exhalation etc. It is further implemented by using various kinds of sensors like IR sensor, Pressure sensor etc. A couple of attributes of the system can be enhanced like signal range and signal strength and by adding the equipment to check and monitor the worker heartbeat and blood pressure.

\section{REFERENCES}

[1] B. Yakub, Epuri Deepthi ,Vivek Jain, Vikram Singh,Ravinder Singh "IOT Mining And worker Safety Helmet" Hyderabad Institute of Technology special issue(NCRCEST) E ISSN:2321-9637 August 2019.

[2] Jagadesh. R ,R. Nagaraja "IOT Based Smart Helmet for Unsafe Event Detection in Mining Industry" Banglore Institute of Technology(IRJET) vol:4 Issue 1, e ISSN:2395-0056 p-ISSN:2395-0072 2017.

[3] T.Pathan,Puja G. Panchbuddhe, Assitant Professor "Micro controller Based Smart Helmet for Coal Miner Safety" Priyadarshini Bhagavati College of Engineering(IJRASET) Vol:6 Issue:4, ISSN-2321-9653 April 2018.

[4] C.T Behr, A. Kumar "A Smart Helmet for Air Quality And Hazardarous Event Detection for Mining industry IEEE 2016.

[5] Sunil E Waghware, Prof. Ashish Manusumare, Prof. Vijay Joy "Micro controller Based Smart Helmet For Coal Miner Safety" Ballarpur Institute f Technology (IJSTR) Vol:6 Issue:8,ISSN-2278-7798 August 2017.

[6] M. Naveen Raj , P.Ashwin Kumar,R. Vignesh, K.Iniyan ,M.Krishna Prasanth Assitant Professor "IOT Based Smart Helmet for Unsafe Detection for Mining Industry" Karapagam College of Engineering(IJPAM.eu) Vol:118 Issue 20,ISSN:533-538 2018.

[7] Shruti P.Bokar,V.B.Baru "IOT Based Smart Helmet for Underground Mines" SCOE,Pune (IJSREM) Vol:1 Issue:9, ISSN:2581-5782 Sept-2018.
[8] Sushrut Rhanjurai, Rakesh Johar, Varnyam Sharma, Abhideep Bhatti "IOT based helmet for coal miner safety" (IJSER) Vol:5 ISSN:2347-3878 May 2017.

[9] https://m.iotgecko.com

[10] https://en.m.wikipedia.org/wiki/Radio-frequency-identif ication

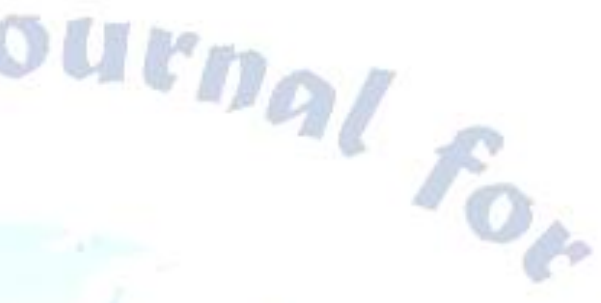

